\title{
The Stochastic Burgers Equation
}

\author{
L. Bertini ${ }^{1,2}$, N. Cancrini ${ }^{1}$, G. Jona-Lasinio ${ }^{1,3}$ \\ 1 Dipartimento di Fisica, Università di Roma "La Sapienza", P.le Aldo Moro 2, 00185 Roma, Italy. \\ E-mail: jona@roma1.infn.it, cancrini@sci.uniroma1.it \\ 2 Dipartimento di Matematica, Università di Roma "Tor Vergata", Via delle Ricerca Scientifica, \\ 00133 Roma, Italy. E-mail: bertini@mat.utovrm.it \\ 3 Centro Linceo Interdisciplinare, Via della Lungaro 10, I-00165 Roma, Italy
}

Received: 21 September 1993

\begin{abstract}
We study Burgers Equation perturbed by a white noise in space and time. We prove the existence of solutions by showing that the Cole-Hopf transformation is meaningful also in the stochastic case. The problem is thus reduced to the anaylsis of a linear equation with multiplicative half white noise. An explicit solution of the latter is constructed through a generalized Feynman-Kac formula. Typical properties of the trajectories are then discussed. A technical result, concerning the regularizing effect of the convolution with the heat kernel, is proved for stochastic integrals.
\end{abstract}

\section{Introduction}

One of the first attempts to arrive at the statistical theory of turbulent fluid motion was the proposal by Burgers of his celebrated equation

$$
\partial_{t} u_{t}(x)=\nu \partial_{x}^{2} u_{t}(x)-u_{t}(x) \partial_{x} u_{t}(x),
$$

where $u_{t}(x)$ is the velocity field and $\nu$ is the viscosity. As Burgers emphasized in the introduction of his book [3] this equation represents an extremely simplified model describing the interaction of dissipative and non-linear inertial terms in the motion of the fluid. A clear discussion on the physical problems connected with Burgers equation can be found in [10]. As shown by Cole and Hopf [5,7], Eq. (1.1) can be explicitly solved and, in the limit of vanishing viscosity, the solution develops shock waves.

Rigorous results have been recently established in the study of some statistical properties: random initial data are considered in $[1,14,16]$, while in [15] a forcing term, which is a stationary stochastic process in time and a periodic function in space, is added.

The study of Burgers equation with a forcing term is interesting in view of the phenomenological character of (1.1). Since it represents an incomplete description of a system, a forcing term can provide a good model of the neglected effects; in particular a random perturbation may help to select interesting invariant measures. 
Translational invariance is preserved when (1.1) is perturbed by additive stochastic processes stationary in space and time.

In principle one can think of a wide variety of stationary random forcing terms. White noise in time and space is very often a candidate and the main motivations behind this choice are a central limit type argument and the insufficient knowledge of the neglected effects or external disturbances. A basic feature of white noise is its singularity at small scales. This may be unphysical in certain cases, but, as stressed in [2], it seems reasonable to expect that when a white noise of small amplitude is added to a deterministic equation the effects of small scales should not be overwhelming in determining the macroscopic behaviour of the system. In other words, using a terminology from quantum field theory, the equation should exhibit some ultraviolet stability. We also note that with this choice, due to the absence of time correlations, the full Galilean invariance of (1.1) is preserved.

In this paper we establish an existence theorem for the Cauchy problem for Burgers equation perturbed by an additive white noise in space and time. Furthermore the theorem gives an explicit expression for the solution. In order to illustrate our result let us write the equation and fix the notations

$$
\partial_{t} u_{t}(x)=\nu \partial_{x}^{2} u_{t}(x)-u_{t}(x) \partial_{x} u_{t}(x)+\varepsilon \eta_{t}(x),
$$

where $t \in \mathbf{R}^{+}, x \in \mathbf{R}, \varepsilon$ is the noise intensity and $\eta_{t}(x)$ is white noise in space and time, i.e.

$$
\mathbf{E}\left(\eta_{t}(x) \eta_{t^{\prime}}\left(x^{\prime}\right)\right)=\delta\left(t-t^{\prime}\right) \delta\left(x-x^{\prime}\right)
$$

We realize the white noise $\eta_{t}(x)$ as the generalized derivative of the brownian sheet, i.e. $\eta_{t}(x)=\partial_{t} \partial_{x} W_{t}(x)$. The gaussian process $W_{t}(x)$ has correlation function

$$
\mathbf{E}^{w}\left(W_{t}(x) W_{t^{\prime}}\left(x^{\prime}\right)\right)=t \wedge t^{\prime} C\left(x, x^{\prime}\right), \quad C\left(x, x^{\prime}\right)=\theta\left(x x^{\prime}\right)|x| \wedge\left|x^{\prime}\right|
$$

where $a \wedge b=\min \{a, b\}$ and $\theta$ is the indicator function of the set $[0, \infty)$. We remark that $C\left(x, x^{\prime}\right)$ is a Lipschitz function.

We write (1.2) as an integral equation using the Green's function of its linear part

$$
u_{t}(x)=G_{t} * u_{0}(x)-\frac{1}{2} \int_{0}^{t} d s G_{t-s}^{\prime} * u_{s}^{2}(x)+\varepsilon \int_{0}^{t} G_{t-s}^{\prime} * d W_{s}(x),
$$

where $*$ is the convolution with the heat kernel

$$
G_{t}(x)=(4 \nu \pi t)^{-1 / 2} \exp \left\{-\frac{x^{2}}{4 \nu t}\right\}
$$

i.e.

$$
G_{t} * f(x)=\int d y G_{t}(x-y) f(y)
$$

and

$$
G_{t}^{\prime} * f(x)=\int d y \partial_{x} G_{t}(x-y) f(y)
$$

finally in (1.5) $d W_{s}(x)$ is the stochastic integral with respect to the brownian sheet and $u_{0}(x)$ is the initial condition. Equation (1.5) is meaningful for a wider class of functions than (1.2), i.e. for functions which do not possess two derivatives. 
We shall construct a solution of Eq. (1.5) by using the Cole-Hopf $[5,7]$ transformation $u_{t}(x)=-2 \nu \partial_{x} \log \psi_{t}(x)$. Proceeding formally it reduces (1.2) to the following linear equation with multiplicative half white noise

$$
\partial_{t} \psi_{t}(x)=\nu \partial_{x}^{2} \psi_{t}(x)-\frac{\varepsilon}{2 \nu} \psi_{t}(x) \partial_{t} W_{t}(x)
$$

In [12] analogous equations, but with white noise, have been studied. Their discrete version have also been considered, e.g. [11].

Burgers equation (1.2) is invariant under translation of the space variable $x$. Due to the presence of the half white noise $\partial_{t} W_{t}(x)$ this property, as can be seen from (1.4), does not hold for (1.9). We show in Sect. 4 how the translation invariance is recovered through the Cole-Hopf transformation.

In order to study rigorously (1.9), one has to interpret it as a Stochastic PDE. Since it contains a non-trivial diffusion the stochastic differential presents the well known ambiguities. In order to obtain, via the Cole-Hopf transformation, the solution of Burgers equation (1.5), the stochastic differential in (1.9) has to be interpreted in the Stratonovich sense [8] as we show in Sect. 4. In the following we thus consider

$$
d \psi_{t}(x)=\nu \partial_{x}^{2} \psi_{t}(x) d t-\frac{\varepsilon}{2 \nu} \psi_{t}(x) \circ d W_{t}(x)
$$

which can be written in terms of the Ito differential as

$$
d \psi_{t}(x)=\left(\nu \partial_{x}^{2} \psi_{t}(x)+\frac{\varepsilon}{2 \nu} V(x) \psi_{t}(x)\right) d t-\frac{\varepsilon}{2 \nu} \psi_{t}(x) d W_{t}(x)
$$

the extra term $V(x)=\frac{1}{2} C(x, x)=\frac{1}{2}|x|$ arises from the formal expression of the Stratonovich differential, see (1.4) above. In this paper we first construct, via a generalized Feynman-Kac formula, a process which solves the mild form of (1.11),

$$
\psi_{t}(x)=G_{t} * \psi_{0}(x)+\frac{\varepsilon}{2 \nu} \int_{0}^{t} G_{t-s} *\left(V \psi_{s} d s-\psi_{s} d W_{s}\right)(x),
$$

where $\psi_{0}(x)$ is the initial condition, it is related to $u_{0}(x)$ by $u_{0}(x)=-2 \nu \partial_{x} \log \psi_{0}(x)$. We then prove that the Cole-Hopf transformation can be applied to $\psi_{t}(x)$ and it defines a solution of (1.5).

In order to give a precise formulation of our results we have to specify in greater detail the mathematical objects appearing in the previous equations.

\section{Preliminaries and Results}

Brownian Sheet. We denote by $\left(\Omega, \mathscr{F}, P^{w}\right)$ the probability space of the brownian sheet $W_{t}(x)$; for its properties see [17]. We introduce the filtration $\mathscr{F}_{t}=$ $\sigma\left\{W_{s}(x):(s, x) \in[0, t] \times \mathbf{R}\right\}$, the minimal $\sigma$-algebra such that $W_{s}(x)$ is measurable for all $s \leq t, x \in \mathbf{R}$.

For Eq. (1.12) we need an integral with respect to the brownian sheet which is an Ito integral in the time variable $t$ and a Bochner integral in the space variable $x$. We thus define, according to Walsh's terminology, the following martingale measure

$$
W_{t}(A)=\int_{A} W_{t}(x) d x, \quad t \in \mathbf{R}^{+}, A \in \mathscr{B}(\mathbf{R}),
$$


where $\mathscr{B}(\mathbf{R})$ is the Borel $\sigma$-algebra of $\mathbf{R}$. As $W_{t}(x)$ is $d P^{w}$-a.s. continuous the above integral is well defined. For every $A, W_{t}(A)$ is a $\mathscr{F}_{t}$ martingale; the cross variation of the martingales $W_{t}(A), W_{t}(B)$ is

$$
\langle W(A), W(B)\rangle_{t}=t \int_{A \times B} d x d x^{\prime} C\left(x, x^{\prime}\right) .
$$

According to Walsh ([17], Chap. II) we can thus define the Ito-Bochner integral with respect to the brownian sheet for $\mathscr{F}_{t}$ progressively measurable functions $f=$ $f(t, x, \omega)$ such that

$$
\mathbf{E}^{w} \int_{0}^{t} d s(f, C f)=\mathbf{E}^{w} \int_{0}^{t} d s \int d x d x^{\prime} C\left(x, x^{\prime}\right) f(s, x, \omega) f\left(s, x^{\prime}, \omega\right)<\infty .
$$

This integral will be denoted by

$$
I_{t}(f)=\int_{0}^{t} \int d x f(s, x, \omega) d W_{s}(x)
$$

it is a $\mathscr{F}_{t}$ martingale with quadratic variation

$$
\langle I(f), I(f)\rangle_{t}=\int_{0}^{t} d s(f, C f) .
$$

In the next sections we use the Burkholder-Davis-Gundy inequality (see e.g. [13], IV 4.2) for the stochastic integral (2.4): for all $p \geq 2$ exists $c_{p}>0$ such that

$$
\left\|I_{t}(f)\right\|_{p} \leq c_{p}\left\|\int_{0}^{t} d s(f, C f)\right\|_{p / 2}^{1 / 2}
$$

where here and throughout the paper $\|\cdot\|_{p}$ is the norm in $L^{p}\left(d P^{w}\right)$. The inequality (2.6) follows from the martingale property and (2.5).

Stochastic Curvilinear Integral. We shall construct a solution of (1.12) via a Feynman-Kac formula. In this formula a Stochastic Curvilinear Integral that we now define will appear. Let $s \mapsto \varphi_{s}$ be a Hölder continuous function from $[0, t]$ to $\mathbf{R}$ and $s_{k}=2^{-n} k t, k=0, \ldots, 2^{n}$ be a partition of $[0, t]$, introduce

$$
M_{\varphi}^{n}(t)=\sum_{k}\left(W_{s_{k+1}}\left(\varphi_{s_{k}}\right)-W_{s_{k}}\left(\varphi_{s_{k}}\right)\right)
$$

using (1.4) we have

$$
\lim _{n \rightarrow \infty} \mathbf{E}^{w}\left(M_{\varphi}^{n}(t)\right)^{2}=\lim _{n \rightarrow \infty} \sum_{k} C\left(\varphi_{s_{k}}, \varphi_{s_{k}}\right)\left(s_{k+1}-s_{k}\right)=\int_{0}^{t} d s\left|\varphi_{s}\right|
$$


which has the geometrical interpretation of the area under the curve $s \mapsto\left|\varphi_{s}\right|$. It is not difficult to verify that $M_{\varphi}^{n}(t)$ is a Cauchy sequence in $L^{2}\left(d P^{w}\right)$; its limit defines

$$
M_{\varphi}(t)=\int_{0}^{t} d W_{s}\left(\varphi_{s}\right), \quad t \in[0, T]
$$

$M_{\varphi}(t)$ is a $d P^{w}$-a.s. continuous gaussian process and a $\mathscr{F}_{t}$ martingale. If $s \mapsto \gamma_{s}$ is another curve the cross variation of $M_{\varphi}(t)$ and $M_{\gamma}(t)$ is

$$
\left\langle M_{\varphi}, M_{\gamma}\right\rangle_{t}=\int_{0}^{t} d s C\left(\varphi_{s}, \gamma_{s}\right)
$$

We note that a weaker form of the integral (2.9) was introduced, in a different context, in [4]. A general theory of stochastic curvilinear integrals is developed in [6]; there the Lipschitz property of the process in the $x$ variable is assumed, therefore our case is not included in that theory.

Results. On the initial condition $u_{0}(x)$ we assume the following. It is a continuous function we write in the form $u_{0}(x)=-\partial_{x} U_{0}(x)$; there exist $a, c>0$ such that for all $x \in \mathbf{R}\left|U_{0}(x)\right| \leq a(1+|x|)$ and $\left|U_{0}^{\prime}(x)\right| \leq c \exp (a|x|)$. These conditions are satisfied when $u_{0}$ is uniformly bounded. They include also some initial data with wild oscillations at infinity, e.g. $u_{0}(x)=e^{x} \sin e^{x}$ is allowed.

We first state the results concerning the linear equation (1.12). We note that the stochastic integral in that equation, according to (2.5), is well defined, in $L^{2}\left(d P^{w}\right)$, if

$$
\begin{aligned}
& \left\|\int_{0}^{t} G_{t-s} *\left(\psi_{s} d W_{s}\right)(x)\right\|_{2}^{2} \\
& =\mathbf{E}^{w} \int_{0}^{t} d s \int d y d y^{\prime} G_{t-s}(x-y) G_{t-s}\left(x-y^{\prime}\right) \\
& \quad \times C\left(y, y^{\prime}\right) \psi_{s}(y) \psi_{s}\left(y^{\prime}\right)<\infty .
\end{aligned}
$$

We now introduce an auxiliary brownian motion which will permit to write a solution of (1.12) as a generalized Feynman-Kac formula. Let $d P_{x, t}^{\beta}=d y d P_{y, 0 ; x, t}^{\beta}$, where $d P_{y, 0 ; x, t}^{\beta}$ is the measure of a backward brownian motion with diffusion coefficient $2 \nu$ starting at time $\mathrm{t}$ in $x$ and arriving at time 0 in $y$. We will denote by $\mathbf{E}_{x, t}^{\beta}$ the expectation with respect to the probability measure $d P_{x, t}^{\beta}$. We stress that the brownian motion $\beta$ is independent from the brownian sheet $W$. Proposition 2.1. Let $\psi_{0}=\exp \left\{\frac{1}{2 \nu} U_{0}(x)\right\}$, the assumptions on $U_{0}$ imply that $\psi_{0} \in$
$C^{1}(\mathbf{R})$ and $\exists c_{1}, c_{2}, a>0$ :

$$
\forall x \in \mathbf{R} \quad c_{1} e^{-a|x|} \leq \psi_{0}(x) \leq c_{2} e^{a|x|}, \quad\left|\psi_{0}^{\prime}(x)\right| \leq c_{2} e^{a|x|} .
$$

Set

$$
\psi_{t}(x)=\mathbf{E}_{x, t}^{\beta}\left(\psi_{0}\left(\beta_{0}\right) e^{-\frac{\varepsilon}{2 \nu} \int_{0}^{t} d W_{s}\left(\beta_{s}\right)}\right),
$$

then $\forall(t, x) \in[0, T] \times \mathbf{R}$, 
(i) $\forall p \geq 1 \psi_{t}(x) \in L^{p}\left(d P^{w}\right)$ and it satisfies condition (2.11).

(ii) $d P^{w}$-a.s. $\psi_{t}(x)$ is a solution of (1.12); $t \mapsto \psi_{t}(x)$ is $d P^{w}$-a.s. locally Hölder continuous with exponent $\alpha<1 / 2$.

(iii) $x \mapsto \psi_{t}(x)$ is $d P^{w}$-a.s. differentiable; its derivative is

$$
\partial_{x} \psi_{t}(x)=G_{t}^{\prime} * \psi_{0}(x)+\frac{\varepsilon}{2 \nu} \int_{0}^{t} G_{t-s}^{\prime} *\left(V \psi_{s} d s-\psi_{s} d W_{s}\right)(x)
$$

$\forall p \geq 1 \partial_{x} \psi_{t}(x) \in L^{p}\left(d P^{w}\right)$. Furthermore the application $(t, x) \mapsto \partial_{x} \psi_{t}(x)$ is $d P^{w_{-}}$ a.s. locally Hölder continuous with exponent $\alpha<1 / 2$ in space and $\alpha<1 / 4$ in time. (iv) $d P^{w}-$ a.s. $\psi_{t}(x)>0$.

The properties (i)-(ii) are statements about the existence of the solution of (1.12), (iii)-(iv) are required to perform the Cole-Hopf transformation.

From Proposition 2.1 we have the following theorem which states the existence result for the stochastic Burgers equation (1.5).

Theorem 2.2. Set $u_{t}(x)=-2 \nu \partial_{x} \log \psi_{t}(x)$, with $\psi_{t}(x)$ given by (2.13).

Then $u_{t}=u_{t}(x)$ is a $C^{0}(\mathbf{R})$-valued, $\mathscr{F}_{t}$-adapted process, such that for all $(t, x) \in$ $[0, T] \times \mathbf{R}$,

(i) $\forall p \geq 1 u_{t}(x) \in L^{p}\left(d P^{w}\right)$; $(t, x) \mapsto u_{t}(x)$ is $d P^{w}$-a.s. locally Hölder continuous with exponent $\alpha<1 / 2$ in space and $\alpha<1 / 4$ in time.

(ii) $u_{t}(x)$ solves $(1.5) d P^{w}$-a.s.

The paper is structured as follows. In the next section we prove a regularizing property for the stochastic convolution with the heat kernel; this is the main technical result in the paper. In Sect. 4 we prove, assuming Proposition 2.1, Theorem 2.2. Proposition 2.1 is then proved in Sect. 5. Moment estimates for $\psi_{t}(x)$ and $\log \psi_{t}(x)$ are obtained in Sect. 6; they give some insight on the behaviour of $u_{t}(x)$ at large $x$. Finally in Sect. 7 some open problems are discussed.

To simplify the notations we assume $\varepsilon=1, \nu=1 / 2$.

\section{Stochastic Convolution with the Heat Kernel}

We here extend to the stochastic case the regularization property of the heat kernel. In particular we show that the brownian sheet integral of the convolution of the heat kernel with a locally Hölder continuous process is $d P^{w}$-a.s. differentiable. The derivative is $d P^{w}$-a.s. locally Hölder continuous. This is the result that permits us to prove the differentiability of $x \mapsto \psi_{t}(x)$ in Proposition 2.1, hence the Cole-Hopf transformation is meaningful also for the stochastic Burgers equation. This result, however, has an independent interest.

Theorem 3.1. Let $\xi_{t}=\xi_{t}(x)$ a continuous $\mathscr{F}_{t}$-adapted process, assume the following properties hold for some $p \geq 2$ :

(a) $\exists c_{1}, a_{1}>0$ such that $\forall x \in \mathbf{R} \sup _{t \in[0, T]}\left\|\xi_{t}(x)\right\|_{p} \leq c_{1} e^{a_{1}|x|}$.

(b) $\exists c_{2}, a_{2}, \alpha>0$ such that

$$
\forall x, y \in \mathbf{R}, \forall t \in[0, T] \quad\left\|\xi_{t}(y)-\xi_{t}(x)\right\|_{p} \leq c_{2} e^{a_{2}(|x|+|y|)}|x-y|^{\alpha} .
$$

Define

$$
F_{t}(x)=\int_{0}^{t} G_{t-s} *\left(\xi_{s} d W_{s}\right)(x)
$$


Then

(i) $x \mapsto F_{t}(x)$ is $L^{p}\left(d P^{w}\right)$ differentiable; its derivative is

$$
\partial_{x} F_{t}(x)=\int_{0}^{t} G_{t-s}^{\prime} *\left(\xi_{s} d W_{s}\right)(x)
$$

and $\exists c_{1}^{\prime}, a_{1}^{\prime}>0$ such that $\forall x \in \mathbf{R} \sup _{t \in[0, T]}\left\|\partial_{x} F_{t}(x)\right\|_{p} \leq c_{1}^{\prime} e^{a_{1}^{\prime}|x|}$.

(ii) $x \mapsto \partial_{x} F_{t}(x)$ is $L^{p}\left(d P^{w}\right)$ locally Hölder continuous with exponent less than $\alpha$, i.e. for any $\varepsilon>0$,

$$
\forall x, y \in \mathbf{R}, \forall t \in[0, T] \quad\left\|\partial_{y} F_{t}(y)-\partial_{x} F_{t}(x)\right\|_{p} \leq c_{2}^{\prime} e^{a_{2}^{\prime}(|x|+|y|)}|x-y|^{\alpha-\varepsilon} .
$$

(iii) If the conditions (a)-(b) are satisfied for all $p<\infty$, in general with $c$ and a p-dependent constants, then $x \mapsto F_{t}(x)$ is also $d P^{w}$-a.s. differentiable; $x \mapsto \partial_{x} F_{t}(x)$ is $d P^{w}$-a.s. Hölder continuous with exponent smaller than $\alpha$.

Proof. (i) We first discuss in detail the case $p=2$. Let us introduce the notations $\Delta_{h} f(x)=h^{-1}(f(x+h)-f(x))$ and $R_{f}(x, h)=\Delta_{h} f(x)-\partial_{x} f(x)$; we will show that

$$
\begin{aligned}
& \left\|\int_{0}^{t} R_{G_{t-s}}(h) *\left(\xi_{s} d W_{s}\right)(x)\right\|_{2}^{2} \\
& =\mathbf{E}^{w} \int_{0}^{t} d s \int d y d y^{\prime} R_{G_{t-s}}(x-y, h) \\
& \quad \times R_{G_{t-s}}\left(x-y^{\prime}, h\right) C\left(y, y^{\prime}\right) \xi_{s}(y) \xi_{s}\left(y^{\prime}\right)
\end{aligned}
$$

converges to 0 when $h \rightarrow 0$. This requires an exchange of the limit $h \rightarrow 0$ with the integrals; due the specificity of this problem we cannot appeal to general theorems but we will estimate explicitly the integral.

Trying to bound directly the integrand in (3.5) a non-integrable singularity $(t-s)^{-1}$ appears. We thus need to exhibit a cancellation.

The key point is that $\int d y R_{G_{t-s}}(x-y, h)=0$, so we can replace the right-hand side of (3.5) by

$$
\int_{0}^{t} d s \int d y d y^{\prime} R_{G_{t-s}}(x-y, h) R_{G_{t-s}}\left(x-y^{\prime}, h\right) \mathbf{E}^{w}\left(\Gamma_{s}\left(x, y, y^{\prime}\right)\right),
$$

where

$$
\Gamma_{s}\left(x, y, y^{\prime}\right)=C\left(y, y^{\prime}\right) \xi_{s}(y) \xi_{s}\left(y^{\prime}\right)-C\left(y^{\prime}, x\right) \xi_{s}\left(y^{\prime}\right) \xi_{s}(x)
$$

We can now use the Hölder continuity assumption. In fact from the hypothesis (a), (b) and the global Lipschitz property for $C\left(y, y^{\prime}\right)$, we get the bound

$$
\sup _{s \in[0, T]} \mathbf{E}^{w}\left|\Gamma_{s}\left(x, y, y^{\prime}\right)\right| \leq c e^{a\left(|y|+\left|y^{\prime}\right|+|x|\right)}|y-x|^{\alpha} .
$$


Let us first consider in (3.6) the integral in $d y^{\prime}$, we show it can be bounded by $(t-s)^{-1 / 2}$. We have

$$
\int_{\left|y^{\prime}-x\right| \leq 2|h|} d y^{\prime} R_{G_{t-s}}\left(x-y^{\prime}, h\right) e^{a\left|y^{\prime}\right|} \leq c e^{a(2|h|+|x|)} \frac{1}{\sqrt{t-s}}
$$

on the other hand, using the Lagrange theorem we get

$$
\begin{aligned}
& \int_{\left|y^{\prime}-x\right|>2|h|} d y^{\prime} \Delta_{h} G_{t-s}\left(x-y^{\prime}\right) e^{a\left|y^{\prime}\right|} \\
& \leq c \int_{|z|>2|h|} d z \frac{|z+\zeta|}{(t-s)^{3 / 2}} e^{-\frac{(z+\zeta)^{2}}{2(t-s)}} e^{a(|z|+|x|)} \\
& \leq c e^{a|x|} \int_{|z|>2|h|} d z \frac{|z|+|h|}{(t-s)^{3 / 2}} e^{-\frac{z^{2}}{8(t-s)}} e^{a|z|} \leq c e^{a|z|} \frac{1}{\sqrt{t-s}}
\end{aligned}
$$

where $|\zeta| \leq|h|$ and thus $|z+\zeta|>\frac{1}{2}|z|$ for $|z|>2|h|$.

We can proceed analogously for the term with $\partial_{x} G_{t-s}(x-y)$.

Using the previous bound and the Hölder property, we now prove that (3.6) vanishes when $h \rightarrow 0$. Due to (3.9) and (3.10) it is enough to show that

$$
\lim _{h \rightarrow 0} \int_{0}^{t} d s \frac{1}{\sqrt{t-s}} \int d y R_{G_{t-s}}(x-y, h) e^{a|y|}|y-x|^{\alpha}=0 .
$$

We consider first the region $|x-y| \leq 2|h|$. To prove (3.11) in this region we show that the integrals arising from each of the three terms in $R_{G}$ converge to zero. We have

$$
\begin{aligned}
& (t-s)^{-1 / 2} \int_{|y-x| \leq 2|h|} d y\left|\partial_{x} G_{t-s}(x-y)\right| e^{a|y|}|y-x|^{\alpha} \\
& \quad \leq c e^{a|x|} \int_{|z| \leq 2|h|} d z \frac{|z|^{1+\alpha}}{(t-s)^{3 / 2}} G_{t-s}(z) \leq c e^{a|x|} h^{\alpha / 2}(t-s)^{-1+\alpha / 4}
\end{aligned}
$$

which is integrable in $d s$. Analogously

$$
\begin{aligned}
& (t-s)^{-1 / 2} \int_{|y-x| \leq 2|h|} d y\left|h^{-1} G_{t-s}(x-y)\right| e^{a|y|}|y-x|^{\alpha} \\
& \quad \leq c e^{a|x|}|h|^{-1+\alpha / 2}(t-s)^{-1+a / 2} \int_{|z| \leq 2|h|} d z \frac{|z|^{\alpha / 2}}{(t-s)^{\alpha / 4}} e^{-\frac{z^{2}}{2(t-s)}} \\
& \quad \leq c e^{a|x|}|h|^{\alpha / 2}(t-s)^{-1+\alpha / 4} .
\end{aligned}
$$

For the last term we use the following estimate on the heat kernel:

$$
G_{t}(z) \leq \frac{c}{\sqrt{t}+|z|}
$$


and integrate explicitly on $d s$. We have

$$
\begin{aligned}
& \int_{0}^{t} d s(t-s)^{-1 / 2} \int_{|y-x| \leq 2|h|} d y|h|^{-1} G_{t-s}(x+h-y) e^{a|y|}|y-x|^{\alpha} \\
& \leq c e^{a|x|} \int_{|z| \leq 2|h|} d z \int_{0}^{t} d s(t-s)^{-1 / 2}|h|^{-1}|z|^{\alpha} \frac{c}{\sqrt{t-s}+|z+h|} \\
& =c e^{a|x|} \int_{|z| \leq 2|h|} d z|h|^{-1}|z|^{\alpha} \log \left(1+\frac{\sqrt{t}}{|z+h|}\right) \leq c e^{a|x|} c(b)|h|^{\alpha-b}
\end{aligned}
$$

for any $b>0$ since the logarithm is bounded by any power. Then it is enough to take $b<\alpha$.

We now consider the case $|x-y|>2|h|$; we show that the integrand is uniformly bounded; we are then allowed to take the limit $h \rightarrow 0$ inside the integrals and (3.11) follows. We have

$$
\begin{aligned}
& (t-s)^{-1 / 2} \int_{|y-x|>2|h|} d y\left|\partial_{x} G_{t-s}(x-y)\right| e^{a|y|}|y-x|^{\alpha} \\
& \quad \leq c e^{a|x|} \int d z \frac{|z|^{1+a}}{(t-s)^{3 / 2}} G_{t-s}(z) e^{a|z|} \leq c e^{a|x|}(t-s)^{-1+\alpha / 2}
\end{aligned}
$$

and, proceeding as in (3.10),

$$
\begin{aligned}
& \int_{\left|y^{\prime}-x\right|>2|h|} d y^{\prime} \Delta_{h} G_{t-s}\left(x-y^{\prime}\right) e^{a\left|y^{\prime}\right|} \\
& \leq c e^{a|x|} \int_{|z|>2|h|} d z \frac{|z|^{1+\alpha}}{(t-s)^{3 / 2}} e^{-\frac{z^{2}}{8(t-s)}} e^{a|z|}
\end{aligned}
$$

(3.16) and (3.17) are integrable in $d s$.

This concludes the proof of the $L^{2}\left(d P^{w}\right)$ differentiability of $x \mapsto F_{t}(x)$.

The case $p>2$ can be treated essentially in the same way. In fact from the B.D.G. inequality (2.6) we get

$$
\begin{aligned}
\left\|\int_{0}^{t} R_{G_{t-s}}(h) *\left(\xi_{s} d W_{s}\right)(x)\right\|_{p} \leq & c(p) \| \int_{0}^{t} d s \int d y d y^{\prime} R_{G_{t-s}}(x-y, h) \\
& \times R_{G_{t-s}}\left(x-y^{\prime}, h\right) C\left(y, y^{\prime}\right) \xi_{s}(y) \xi_{s}\left(y^{\prime}\right) \|_{p / 2}^{1 / 2} \\
\leq & c(p)\left[\int_{0}^{t} d s \int d y d y^{\prime}\left|R_{G_{t-s}}(x-y, h)\right|^{1 / 2}\right. \\
& \left.\times\left|R_{G_{t-s}}\left(x-y^{\prime}, h\right)\right|\left\|\Gamma_{s}\left(x, y, y^{\prime}\right)\right\|_{p / 2}\right]^{1 /}
\end{aligned}
$$


The proof now continues as for $p=2$. We remark that, due to the explicit dependence on $t$ of the heat kernel, $F_{t}(x)$ defined in (3.2) is not a martingale; however the B.D.G. inequality holds as follows from the following argument. Consider first

$$
\int_{0}^{t} G_{t_{1}-s} *\left(\xi_{s} d W_{s}\right)(x) \text { for } t<t_{1}
$$

which is a $\mathscr{F}_{t}$ martingale; apply the B.D.G. inequality and then let $t \rightarrow t_{1}$.

(ii) It follows exactly the same steps of (i), but with $\Delta_{h} G_{t-s}(x-y)-\partial_{x} G_{t-s}(x-y)$ in (3.5) replaced by $\partial_{x} G_{t-s}(x+h-y)-\partial_{x} G_{t-s}(x-y)$.

(iii) From the above discussion we have

$$
\forall p \geq 1 \quad \exists c: \forall \varepsilon>0 \quad\left\|\Delta_{h} \psi_{t}(x)-\partial_{x} \psi_{t}(x)\right\|_{p} \leq c h^{\alpha-\varepsilon}
$$

the $d P^{w}$-a.s. differentiability follows then from Kolmogorov Theorem (see e.g. [13], 12.1). Analogously the $d P^{w}$-a.s. Hölder continuity of $x \mapsto \partial_{x} F_{t}(x)$ follows from (ii).

\section{Cole-Hopf Transformation and Ito Calculus}

In this section we show how Theorem 2.2 follows from Proposition 2.1. In order to verify that the process $u_{t}(x)=-\partial_{x} \log \psi_{t}(x)$ satisfies Burgers equation (1.5) we introduce a regularization. This procedure is needed to apply stochastic calculus. After the regularization is removed we obtain a mild form of the Ito formula for a function of the process $\psi_{t}(x)$. Finally we show how translation invariance for Burgers equation is recovered via the Cole-Hopf transformation.

Proof of Theorem 2.2. (i) From Jensen inequality and (2.13) we have

$$
\frac{1}{\psi_{t}(x)}=\frac{1}{\mathbf{E}_{x, t}^{\beta}\left(\psi_{0}\left(\beta_{0}\right) e^{-\int_{0}^{t} d W_{s}\left(\beta_{s}\right)}\right)} \leq \mathbf{E}_{x, t}^{\beta}\left(\psi_{0}\left(\beta_{0}\right)^{-1} e^{\int^{t} d W_{s}\left(\beta_{s}\right)}\right) .
$$

As it will be done in the proof of (i) in Proposition 2.1 it can be verified that the righthand side of (4.1) is in $L^{p}\left(d P^{w}\right)$. From this and (iii) of Proposition 2.1 it follows that $u_{t}(x) \in L^{p}\left(d P^{w}\right)$. The Hölder continuity follows directly from statements (ii)-(iv) in Proposition 2.1.

(ii) Let us introduce a regularization of the brownian sheet

$$
W_{s}^{\kappa}(x)=\delta^{\kappa} * W_{s}(x)=\int d y \delta^{\kappa}(x-y) W_{s}(y)
$$

where $\delta^{\kappa}(x)=\kappa h(\kappa x)$ with $h \in C_{0}^{\infty}(\mathbf{R})$ a smooth positive function with compact support and $\int d x h(x)=1$. The covariance of the process (4.2) is $t \wedge t^{\prime} C^{\kappa}\left(x, x^{\prime}\right)$, where $C^{\kappa}=\delta^{\kappa} * C * \delta^{\kappa}$. Correspondingly we have $\psi_{t}^{\kappa}(x)$ which satisfies the regularized version of (1.11), i.e.

$$
\begin{aligned}
d \psi_{t}^{\kappa}(x) & =\left(\frac{1}{2} \partial_{x}^{2} \psi_{t}^{\kappa}(x)+V^{\kappa}(x) \psi_{t}^{\kappa}(x)\right) d t-\psi_{t}^{\kappa}(x) d W_{t}^{\kappa}(x) \\
\psi_{t=0}^{\kappa}(x) & =\psi_{0}(x)
\end{aligned}
$$

where $V^{\kappa}(x)=\frac{1}{2} C^{\kappa}(x, x)$. We remark that the second derivative with respect to $x$ is now meaningful. 
Let us establish the convergence of the regularized process:

$$
\lim _{\kappa \rightarrow \infty}\left\|\psi_{t}^{\kappa}(x)-\psi_{t}(x)\right\|_{p}=0
$$

Using the Feynman-Kac representation (2.13) and denoting by $M_{\beta}^{\kappa}(t)$ the curvilinear stochastic integral for the regularized brownian sheet we have

$$
\begin{aligned}
& \left\|\psi_{t}^{\kappa}(x)-\psi_{t}(x)\right\|_{p} \\
& \quad \leq\left\|\mathbf{E}_{x, t}^{\beta}\left(\psi_{0}\left(\beta_{0}\right)\left(e^{-M_{\beta}^{\kappa}(t)}+e^{-M_{\beta}(t)}\right)\left|M_{\beta}^{\kappa}(t)-M_{\beta}(t)\right|\right)\right\|_{p} \\
& \quad \leq \mathbf{E}_{x, t}^{\beta}\left(\psi_{0}\left(\beta_{0}\right)\left\|e^{-M_{\beta}^{\kappa}(t)}+e^{-M_{\beta}(t)}\right\|_{2 p}\left\|M_{\beta}^{\kappa}(t)-M_{\beta}(t)\right\|_{2 p}\right) .
\end{aligned}
$$

From the bound $\left|C * \delta^{\kappa}(x, y)-C(x, y)\right| \leq c \kappa^{-1}$ we see that the second factor converges, uniformly in $\beta$, to 0 as $\kappa \rightarrow \infty$. From Proposition 2.1, the first factor is bounded uniformly in $\kappa$.

Using the expression (2.14) for $\partial_{x} \psi_{t}(x)$ and following the same argument of Theorem 3.1, it is easy to prove also the $L^{p}\left(d P^{w}\right)$ convergence of the derivative, i.e.

$$
\lim _{\kappa \rightarrow \infty}\left\|\partial_{x} \psi_{t}^{\kappa}(x)-\partial_{x} \psi_{t}(x)\right\|_{p}=0
$$

Let $u_{t}^{\kappa}(x)=-\partial_{x} \log \psi_{t}^{\kappa}(x)$ : we show it satisfies

$$
u_{t}^{\kappa}(x)=G_{t} * u_{0}(x)-\frac{1}{2} \int_{0}^{t} d s G_{t-s}^{\prime} *\left(u_{s}^{\kappa}\right)^{2}(x)+\int_{0}^{t} G_{t-s}^{\prime} * d W_{s}^{\kappa}(x) .
$$

Integrating by parts we have the following identity:

$$
\begin{aligned}
& \int_{0}^{t} d s G_{t-s} *\left(\frac{\partial^{2} \psi_{s}^{\kappa}}{\psi_{s}^{\kappa}}\right)(x) \\
& \quad=\int_{0}^{t} d s G_{t-s}^{\prime} *\left(\frac{\partial \psi_{s}^{\kappa}}{\psi_{s}^{\kappa}}\right)(x)+\int_{0}^{t} d s G_{t-s} *\left(\frac{\partial \psi_{s}^{\kappa}}{\psi_{s}^{\kappa}}\right)^{2}(x),
\end{aligned}
$$

where $\partial$ means the derivative with respect to the integrated space variable.

Using (4.3) it is a simple Ito calculus exercise to compute the stochastic differential (with respect to $s$ ) of $G_{t-s}(x-y) \log \psi_{s}^{\kappa}(y)$. By (4.8) one then obtains

$$
\begin{aligned}
\log \psi_{t}^{\kappa}(x)= & G_{t} * \log \psi_{0}(x)+\frac{1}{2} \int_{0}^{t} d s G_{t-s} *\left(\frac{\partial \psi_{s}^{\kappa}}{\psi_{s}^{\kappa}}\right)^{2}(x) \\
& -\int_{0}^{t} G_{t-s} * d W_{s}^{\kappa}(x) .
\end{aligned}
$$

Differentiating the above identity we verify that (4.7) is satisfied. We stress that the equation satisfied by $u_{t}^{\kappa}(x)$, defined through the Cole-Hopf transformation, would not have been (4.7) if we had interpreted (1.9) in the Ito sense.

We note that from (4.4) and (4.6) the $L^{p}\left(d P^{w}\right)$ convergence of the right-hand side of (4.8) follows, as $\kappa \rightarrow \infty$. That limit defines $\partial_{x}^{2} \psi_{s}(x) / \psi_{s}(x)$ when convoluted with $G_{t-s}$. In fact, whereas $\partial_{x} \psi_{t}(x)$ exists, as stated in Proposition 2.1, the second 
derivative of $\psi_{t}(x)$ does not have a meaning in the classical sense. Moreover each term in (4.9) converges to the non-regularized one. In the limit $\kappa \rightarrow \infty$ (4.9) is then a mild Ito formula.

In order to complete the proof of Theorem 2.2 we now take the limit $\kappa \rightarrow \infty$ in (4.7) and show that each term converges, in $L^{2}\left(d P^{w}\right)$, to the corresponding nonregularized one in (1.5).

Let us discuss first the stochastic integrals. Along the same line of Theorem 3.1 it can be easily proved that

$$
\lim _{\kappa \rightarrow \infty}\left\|\int_{0}^{t} G_{t-s}^{\prime} *\left(d W_{s}^{\kappa}-d W_{s}\right)(x)\right\|_{2}=0
$$

We now consider

$$
\begin{aligned}
\left\|u_{t}(x)-u_{t}^{\kappa}(x)\right\|_{2} \leq & \left\|\frac{\partial_{x} \psi_{t}^{\kappa}(x)}{\psi_{t}(x) \psi_{t}^{\kappa}(x)}\right\|_{4}\left\|\psi_{t}(x)-\psi_{t}^{\kappa}(x)\right\|_{4} \\
& +\left\|\frac{1}{\psi_{t}(x)}\right\|_{4}\left\|\partial_{x} \psi_{t}(x)-\partial_{x} \psi_{t}^{\kappa}(x)\right\|_{4},
\end{aligned}
$$

which converges to 0 as $\kappa \rightarrow \infty$ by (4.4), (4.6) and (i).

For the last term we have

$$
\begin{aligned}
& \left\|\int_{0}^{t} G_{t-s}^{\prime} *\left(\left(u_{s}^{\kappa}\right)^{2}-\left(u_{s}\right)^{2}\right)(x)\right\|_{2} \\
& \quad \leq \int_{0}^{t} d s \int d y\left|\partial_{x} G_{t-s}(x-y)\right|\left\|u_{s}^{\kappa}(y)+u_{s}(y)\right\|_{4}\left\|u_{s}^{\kappa}(y)-u_{s}(y)\right\|_{4}
\end{aligned}
$$

and $\left\|u_{s}^{\kappa}(y)-u_{s}(y)\right\|_{4}$ can be bounded as in (4.11) by a uniformly integrable function vanishing in the limit $\kappa \rightarrow \infty$.

This implies statement (ii) of the theorem: let us write Eq. (4.7) and (1.5) in the form $F^{\kappa}\left(u^{\kappa}\right)=0$ and $F(u)=0$. We have

$$
\|F(u)\|_{2} \leq\left\|F(u)-F^{\kappa}\left(u^{\kappa}\right)\right\|_{2}+\left\|F^{\kappa}\left(u^{\kappa}\right)\right\|_{2} ;
$$

the first term on the right-hand side vanishes as $\kappa \rightarrow \infty$, the second one is identically 0 .

Translation Invariance. We conclude this section showing how translation invariance for Burgers equation (1.5) is recovered. Let us define the brownian sheet with respect to a point $x=a$ instead of $x=0$, i.e. $W_{t}^{a}(x)=W_{t}(x)-W_{t}(a)$, whose covariance is

$$
\mathbf{E}^{w^{a}}\left(W_{t}^{a}(x) W_{t^{\prime}}^{a}\left(x^{\prime}\right)\right)=t \wedge t^{\prime} \theta\left((x-a)\left(x^{\prime}-a\right)\right)|x-a| \wedge\left|x^{\prime}-a\right| .
$$

The solution of (1.12) with $W_{t}(x)$ replaced by $W_{t}^{a}(x)$ is then

$$
\psi_{t}^{a}(x)=\mathbf{E}_{x, t}^{\beta}\left(\psi_{0}\left(\beta_{0}\right) e^{-\int_{0}^{t} d W_{s}^{a}\left(\beta_{s}\right)}\right)=e^{-\int_{0}^{t} d W_{s}(a)} \mathbf{E}_{x, t}^{\beta}\left(\psi_{0}\left(\beta_{0}\right) e^{-\int_{0}^{t} d W_{s}\left(\beta_{s}\right)}\right) .
$$

In the above expression the dependence on $a$ is factorized, so that, when the Cole-Hopf transformation is performed, it cancels out completely. We remark that the choice of $a$ is related to the possibility to multiply $\psi_{t}(x)$ by an arbitrary time dependent factor which disappears when (4.9) is differentiated to obtain (4.7). 


\section{Solution of the Linear Equation}

In this section we study the linear equation with multiplicative half white noise (1.11); we prove the existence of a solution for its mild form (1.12). This solution is constructed explicitly through a generalized Feynman-Kac formula (2.13). We then study the typical properties of the trajectories: they are strictly positive and differentiable with respect to $x$. The last property is a direct application of Theorem 3.1.

Proof of Proposition 2.1. (i) It is enough to assume $p \in \mathbf{N}$. Let $\vec{\beta}=\left\{\beta^{i}\right\}_{\imath=1}^{n}$ be a family of $n$ independent brownian motions; then, under $d P^{w},\left\{M_{\beta^{i}}(t)\right\}_{i=1}^{n}$ are mean zero Gaussian processes with covariance matrix given by

$$
\mathbf{E}^{w}\left(M_{\beta^{2}}(t) M_{\beta^{\jmath}}\left(t^{\prime}\right)\right)=\int_{0}^{t \wedge t^{\prime}} d s C\left(\beta_{s}^{\imath}, \beta_{s}^{j}\right) .
$$

This is a direct consequence of the gaussian property of the system $\left\{M_{\beta^{\imath}}(t)\right\}_{i=1}^{n}$ and (2.10).

We have

$$
\begin{aligned}
\left.\| \psi_{t}(x)\right) \|_{p}^{p} & =\left\|\prod_{i=1}^{p} \mathbf{E}_{x, t}^{\beta^{2}}\left(\psi_{0}\left(\beta_{0}^{\imath}\right) e^{-M_{\beta^{i}}(t)}\right)\right\|_{p}^{p}=\mathbf{E}_{x, t}^{\vec{\beta}} \mathbf{E}^{w}\left(\prod_{\imath=1}^{p} \psi_{0}\left(\beta_{0}^{i}\right) e^{-M_{\beta^{\imath}}(t)}\right) \\
& =\mathbf{E}_{x, t}^{\vec{\beta}}\left(\prod_{i=1}^{p} \psi_{0}\left(\beta_{0}^{i}\right) \exp \left\{\frac{1}{2} \sum_{i, j} \int_{0}^{t} d s C\left(\beta_{s}^{\imath}, \beta_{s}^{\jmath}\right)\right\}\right) \\
& \leq c_{2}^{p}\left[\mathbf{E}_{0, t}^{\beta}\left(\exp \left\{a\left|x+\beta_{0}\right|+\frac{p}{2} \int_{0}^{t} d s\left|x+\beta_{s}\right|\right\}\right)\right]^{p} \\
& \leq c_{2}^{p} \exp \left\{\frac{1}{2} p^{2}|x| t+a p|x|+\frac{1}{4 !}\left((p t+2 a)^{3}-(2 a)^{3}\right)\right\} .
\end{aligned}
$$

From the above bound it is easy to check that condition (2.11) is satisfied.

(ii) We now verify that $\psi_{t}(x)$ given by the Feynman-Kac formula (2.13) is the solution of (1.12). We have

$$
\begin{aligned}
& \int_{0}^{t} G_{t-s} *\left(V \psi_{s} d s-\psi_{s} d W_{s}\right)(x) \\
& \quad=\int_{0}^{t} \int d y G_{t-s}(x-y) \mathbf{E}_{s, y}^{\beta}\left(\psi_{0}\left(\beta_{0}\right) e^{-M_{\beta}(s)}\right)\left(V(y) d s-d W_{s}(y)\right),
\end{aligned}
$$

the kernel $G_{t-s}(x-y)$ can be interpreted as the transition probability of the brownian motion $\beta_{s}$. Hence, using the Markov property, (5.3) equals

$$
\mathbf{E}_{x, t}^{\beta}\left(\psi_{0}\left(\beta_{0}\right) \int_{0}^{t} e^{-M_{\beta}(s)}\left(V\left(\beta_{s}\right) d s-d W_{s}\left(\beta_{s}\right)\right)\right) .
$$


Computing the stochastic differential (with respect to $W$ ) of $\exp \left\{-M_{\beta}(s)\right\}$ we get

$$
\begin{aligned}
d\left(e^{-M_{\beta}(s)}\right) & =e^{-M_{\beta}(s)}\left(-d M_{\beta}(s)+\frac{1}{2} d\left\langle M_{\beta}, M_{\beta}\right\rangle_{s}\right) \\
& =e^{-M_{\beta}(s)}\left(-d W_{s}\left(\beta_{s}\right)+V\left(\beta_{s}\right) d s\right)
\end{aligned}
$$

Thus (5.4) is equal to

$$
\mathbf{E}_{x, t}^{\beta}\left(\psi_{0}\left(\beta_{0}\right)\left(e^{-\int_{0}^{t} d W_{s}\left(\beta_{s}\right)}-1\right)\right)=\psi_{t}(x)-G_{t} * \psi_{0}(x),
$$

and Eq. (1.12) is verified.

We now discuss the continuity of $(t, x) \mapsto \psi_{t}(x)$. Using the Feynman-Kac representation (2.13), we prove the following bound. For any $p \geq 1, \exists c, a>0$ such that $\forall t \in[0, T]$

$$
\left\|\psi_{t}(x)-\psi_{t}(y)\right\|_{p} \leq c e^{a(|x|+|y|)}|x-y|^{1 / 2}
$$

From the triangular and Cauchy-Schwartz inequalities we get

$$
\begin{aligned}
& \left\|\psi_{t}(x)-\psi_{t}(y)\right\|_{p} \leq\left\|\mathbf{E}_{0, t}^{\beta}\left(\left(\psi_{0}\left(\beta_{0}+x\right)-\psi_{0}\left(\beta_{0}+y\right)\right) e^{\left.-M_{\beta+x}(t)\right)}\right)\right\|_{p} \\
& \quad+\left\|\mathbf{E}_{0, t}^{\beta}\left(\psi_{0}\left(\beta_{0}+y\right)\left(e^{-M_{\beta+x}(t)}+e^{-M_{\beta+y}(t)}\right)\left|M_{\beta+x}(t)-M_{\beta+y}(t)\right|\right)\right\|_{p} \\
& \leq \\
& \quad c e^{a(|x|+|y|)}|y-x| \\
& \quad+\mathbf{E}_{0, t}^{\beta}\left(\psi_{0}\left(\beta_{0}+y\right)\left\|e^{-M_{\beta+x}(t)}+e^{-M_{\beta+y}(t)}\right\|_{2 p}\right. \\
& \left.\quad \times\left\|M_{\beta+x}(t)-M_{\beta+y}(t)\right\|_{2 p}\right)
\end{aligned}
$$

on the other hand, $\left\|M_{\beta+x}(t)-M_{\beta+y}\right\|_{2 p}$ can be bounded by

$$
\begin{aligned}
& c\left[\int_{0}^{t} d s\left(C\left(\beta_{s}+x, \beta_{s}+x\right)-2 C\left(\beta_{s}+x, \beta_{s}+y\right)+C\left(\beta_{s}+y, \beta_{s}+y\right)\right)\right]^{1 / 2} \\
& \quad \leq c t^{1 / 2}|x-y|^{1 / 2}
\end{aligned}
$$

Finally (5.7) follows from (5.2), (5.8) and (5.9).

From (1.12), we can obtain

$$
\left\|\psi_{t}(x)-\psi_{s}(x)\right\|_{p} \leq c e^{a|x|}|t-s|^{1 / 2}
$$

We do not prove (5.10) because in the proof of (iii) an analogous, but technically harder, estimate for $\partial_{x} \psi_{t}(x)$ will be obtained.

Using the Kolmogorov Theorem, from (5.7) and (5.10), it follows the $d P^{w}$-a.s. local Hölder continuity of exponent $\alpha<1 / 2$ for $(t, x) \mapsto \psi_{t}(x)$.

(iii) We now show, using (1.12), that $x \mapsto \psi_{t}(x)$ is $L^{p}\left(d P^{w}\right)$ and $d P^{w}$-a.s. differentiable; the derivative is given by $(2.14)$. 
Let $\Delta_{h} f(x)=h^{-1}(f(x+h)-f(x))$, then from (1.12) we get

$$
\Delta_{h} \psi_{t}(x)=\Delta_{h} G_{t} * \psi_{0}(x)+\int_{0}^{t} \Delta_{h} G_{t-s} *\left(V \psi_{s} d s-\psi_{s} d_{s} W\right)(x)
$$

and, for $\partial_{x} \psi_{t}(x)$ given by (2.14), we have

$$
\begin{aligned}
\left\|\Delta_{h} \psi_{t}(x)-\partial_{x} \psi_{t}(x)\right\|_{p} \leq & \left|\left(\Delta_{h} G_{t}-G_{t}^{\prime}\right) * \psi_{0}(x)\right| \\
& +\left\|\int_{0}^{t} d s\left(\Delta_{h} G_{t-s}-G_{t-s}^{\prime}\right) * \psi_{s}(x)\right\|_{p} \\
& +\left\|\int_{0}^{t}\left({ }_{h} G_{t-s}-G_{t-s}^{\prime}\right) *\left(\psi_{s} d W_{s}\right)(x)\right\|_{p} .
\end{aligned}
$$

From the assumptions on the initial condition $\psi_{0}$ we have

$$
\lim _{h \rightarrow 0} \Delta_{h} G_{t} * \psi_{0}(x)=G_{t}^{\prime} * \psi_{0}(x) .
$$

In Theorem 3.1 we showed in detail the convergence to zero of the last term in (5.12); the second one is easier.

Let us discuss the continuity property of the derivative. From (3.4), repeating the above discussion, it is easy to see that $x \mapsto \partial_{x} \psi_{t}(x)$ is $L^{p}\left(d P^{w}\right)$ locally Hölder continuous with exponent $\alpha<1 / 4$.

This implies for $x \mapsto \psi_{t}(x)$ a better estimate than (5.7): it is locally Lipschitz, i.e.

$$
\left\|\psi_{t}(x)-\psi_{t}(y)\right\|_{p} \leq c e^{a(|x|+|y|)}|x-y|
$$

Applying again Theorem 3.1 with this bound instead of (5.7) we prove that $x \mapsto$ $\partial_{x} \psi_{t}(x)$ is $L^{p}\left(d P^{w}\right)$ locally Hölder continuous with exponent $\alpha<1 / 2$,

$$
\left\|\partial_{x} \psi_{t}(x)-\partial_{y} \psi_{t}(y)\right\|_{p} \leq c e^{a(|x|+|y|)}|x-y|^{\frac{1}{2}-\varepsilon} .
$$

We now discuss the $L^{p}\left(d P^{w}\right)$ Hölder continuity of $t \mapsto \partial_{x} \psi_{t}(x)$. The bound (5.10) of (ii) is obtained in the same way. We consider $p=2$, the general case follows via the B.D.G. inequality as in Theorem 3.1.

Let $k>0$, from (2.14) we have, omitting the dependence on the space variable $x$,

$$
\begin{aligned}
\left\|\partial_{x} \psi_{t+k}-\partial_{x} \psi_{t}\right\|_{2} \leq & \left|\left(G_{t+k}^{\prime}-G_{t}^{\prime}\right) * \psi_{0}\right| \\
& +\left\|\int_{0}^{t+k} d s G_{t+k-s}^{\prime} *\left(\psi_{s} V\right)-\int_{0}^{t} d s G_{t-s}^{\prime} *\left(\psi_{s} V\right)\right\|_{2} \\
& +\left\|\int_{0}^{t+k} G_{t+k-s}^{\prime} *\left(\psi_{s} d W_{s}\right)-\int_{0}^{t} G_{t-s}^{\prime} *\left(\psi_{s} d W_{s}\right)\right\|_{2}
\end{aligned}
$$


We consider in detail just the term with the stochastic integral in (5.16); we have

$$
\begin{aligned}
& \left\|\int_{0}^{t+k} G_{t+k-s}^{\prime} *\left(\psi_{s} d W_{s}\right)-\int_{0}^{t} G_{t-s}^{\prime} *\left(\psi_{s} d W_{s}\right)\right\|_{2} \\
& \leq\left\|\int_{t}^{t+k} G_{t+k-s}^{\prime} *\left(\psi_{s} d W_{s}\right)\right\|_{2}+\left\|\int_{t-k}^{t}\left(G_{t+k-s}^{\prime}-G_{t-s}^{\prime}\right) *\left(\psi_{s} d W_{s}\right)\right\|_{2} \\
& \quad+\left\|\int_{0}^{t-k}\left(G_{t+k-s}^{\prime}-G_{t-s}^{\prime}\right) *\left(\psi_{s} d W_{s}\right)\right\|_{2},
\end{aligned}
$$

computing the $L^{2}\left(d P^{w}\right)$ norm, subtracting, as in (3.6), the appropriate null term

$$
\mathbf{E}^{w} \int_{t}^{t+k} d s \int d y d y^{\prime} \partial_{x} G_{t+k-s}(x-y) \partial_{x} G_{t+k-s}\left(x-y^{\prime}\right) C\left(y^{\prime}, x\right) \psi_{s}\left(y^{\prime}\right) \psi_{s}(x)
$$

and using the Lipschitz property for $x \mapsto \psi_{t}(x)$, the first term in (5.17) can be bounded by

$$
\begin{gathered}
c e^{a|x|}\left[\int_{t}^{t+k} d s \int d z d z^{\prime}\left|\partial_{z} G_{t+k-s}(z)\right|\left|\partial_{z^{\prime}} G_{t+k-s}\left(z^{\prime}\right)\right||z| e^{a\left(|z|+\left|z^{\prime}\right|\right)}\right]^{1 / 2} \\
\leq c e^{a|x|}\left[\int_{t}^{t+k} d s(t+k-s)^{-1 / 2}\right]^{1 / 2} \leq c e^{a|x|} k^{1 / 4}
\end{gathered}
$$

The second term in (5.17) is analogous, for the third term we have

$$
\int d z^{\prime}\left|\partial_{z^{\prime}} G_{t+k-s}\left(z^{\prime}\right)-\partial_{z^{\prime}} G_{t-s}\left(z^{\prime}\right)\right| e^{a\left|z^{\prime}\right|} \leq c(t-s)^{-1 / 2}
$$

and using Lagrange theorem

$$
\begin{aligned}
& \int_{z^{2}>k} d z\left|\partial_{z} G_{t+k-s}(z)-\partial_{z} G_{t-s}(z)\right||z| e^{a|z|} \\
& \quad \leq c \int_{z^{2}>k} d z k \frac{z^{2}}{(t-s+\sigma)^{5 / 2}}\left(3+\frac{z^{2}}{t-s+\sigma}\right) e^{-\frac{z^{2}}{2(t-s+\sigma)} e^{a|z|}} \\
& \quad \leq c k^{\frac{1}{2}-\varepsilon} \int d z|z|^{3+2 \varepsilon}(t-s)^{-2}\left(3+\frac{z^{2}}{t-s}\right) G_{2(t-s)}(z) e^{a|z|} \\
& \leq c k^{\frac{1}{2}-\varepsilon}(t-s)^{-\frac{1}{2}+\varepsilon}
\end{aligned}
$$


as $\sigma \in[0, k]$ and $t-s \geq k$. On the other hand,

$$
\begin{aligned}
& \int_{z^{2} \leq k} d z\left|\partial_{z} G_{t+k-s}(z)-\partial_{z} G_{t-s}(z)\right||z| e^{a|z|} \\
& \quad \leq c k^{\frac{1}{2}-\varepsilon} \int d z|z|^{1+2 \varepsilon}\left(\frac{1}{t+k-s} G_{t+k-s}(z)+\frac{1}{t-s} G_{t-s}(z)\right) e^{a|z|} \\
& \quad \leq c k^{\frac{1}{2}-\varepsilon}(t-s)^{-\frac{1}{2}+\varepsilon}
\end{aligned}
$$

hence (5.17) is bounded by $c \exp (a|x|) k^{\frac{1}{4}-\varepsilon}$.

We have thus proved

$$
\begin{gathered}
\forall p \geq 1, \exists c>0: \forall \varepsilon>0 \\
\left\|\partial_{x} \psi_{t+k}(x+h)-\partial_{x} \psi_{t}(x)\right\|_{p} \leq c e^{a|x|}\left(\sqrt{h^{2}+k^{2}}\right)^{\frac{1}{4}-\varepsilon} .
\end{gathered}
$$

From the Kolmogorov Theorem follows the $d P^{w}$-a.s. local Hölder continuity of $(t, x) \mapsto \partial_{x} \psi_{t}(x)$. The Hölder exponent is $\alpha<1 / 4$ in time and $\alpha<1 / 2$ in space.

(iv) We use the expression of $\psi_{t}(x)$ given by the Feynman-Kac formula (2.13). From the hypotheses on $\psi_{0}$ and Jensen inequality we have

$$
\psi_{t}(x) \geq c_{1} \exp \left\{-\mathbf{E}_{x, t}^{\beta}\left(a\left|\beta_{0}\right|+\int_{0}^{t} d W_{s}\left(\beta_{s}\right)\right)\right\}
$$

as

$$
\mathbf{E}^{w}\left(\mathbf{E}_{x, t}^{\beta}\left(\int_{0}^{t} d W_{s}\left(\beta_{s}\right)\right)\right)^{2}<\infty ;
$$

the exponent on the right-hand side of (5.19) is $d P^{w}$-a.s. finite, hence $\psi_{t}(x)>0$ $d P^{w}$-a.s.

\section{Moment Estimates}

In this section we conclude the analysis for the moments of $\psi_{t}(x)$ initiated in Sect. 5 . We obtain a bound from below. We then estimate the moments of $\log \psi_{t}(x)$ which grow slower. They will give some insight on the behavior of $u_{t}(x)$ for large $x$.

Moment estimates for $\psi_{t}(x)$

Proposition 6.1. Let $\psi_{t}(x)$ as in Proposition 2.1, $p \in \mathbf{N}$; then

$$
\begin{aligned}
& c_{1}^{p} e^{\frac{1}{2} p^{2}|x| t-a p|x|+\frac{1}{4 !}\left((p t-2 a)^{3}-(2 a)^{3}\right)} e^{-c p^{2} t^{3 / 2}} \\
& \quad \leq\left\|\psi_{t}(x)\right\|_{p}^{p} \leq c_{2}^{p} e^{\frac{1}{2} p^{2}|x| t+a p|x|+\frac{1}{4 !}\left((p t+2 a)^{3}-(2 a)^{3}\right)}
\end{aligned}
$$

where $a$ is the constant appearing in (2.12).

Proof. Formula (5.2) gives the estimate from above of the $p^{\text {th }}$ moment of $\psi$. We now estimate from below $\|\psi\|_{p}^{p}$. We assume $\psi_{0}(x) \geq c>0$, i.e. $U_{0}(x)$ uniformly bounded and we obtain (6.1) with $a=0$. The general case is only more tedious. 
From (5.2) we have

$$
\begin{aligned}
\| \psi(t, x)) \|_{p}^{p} & =\mathbf{E}_{x, t}^{\vec{\beta}}\left(\psi_{0}\left(\beta_{0}^{1}\right) \ldots \psi_{0}\left(\beta_{0}^{p}\right) \exp \left\{\frac{1}{2} \int_{0}^{t} d s \sum_{\imath, j} C\left(\beta_{s}^{\imath}, \beta_{s}^{j}\right)\right\}\right) \\
& \geq c^{p} \mathbf{E}_{x, t}^{\vec{\beta}}\left(\exp \left\{\frac{p}{2} \int_{0}^{t} d s \sum_{\imath=1}^{p} \beta_{s}^{\imath}-\int_{0}^{t} d s \sum_{1 \leq \imath<\jmath \leq p}\left|\beta_{s}^{i}-\beta_{s}^{\jmath}\right|\right\}\right) .
\end{aligned}
$$

We introduce the brownian motion $\gamma_{s}=\frac{1}{\sqrt{p}} \sum_{i=1}^{p} \beta_{s}^{2}$. The first term in the exponent of (6.2) is the integral of $\gamma_{s}$, the second one is independent on it; the expectation thus factorizes and we get

$$
\| \psi(t, x)) \|_{p}^{p} \geq c_{1}^{p} e^{\frac{1}{2} p^{2}|x| t+\frac{1}{4 !} p^{3} t^{3}} e^{-c p^{2} t^{3 / 2}}
$$

These results do not imply intermittence in the sense of [11] because the field is not spatially ergodic.

Moment estimates for $\log \psi_{t}(x)$

We now study the moments of $\log \psi_{t}(x)$. We start with the estimate from above.

Proposition 6.2. Let $\psi_{t}(x)$ as in Proposition 2.1, then for either $t$ or $|x|$ large enough (i) for $p=2 n+1, n \in \mathbf{N}$,

$$
\mathbf{E}^{w}\left(\log ^{p} \psi_{t}(x)\right) \leq\left[c_{1}+\frac{1}{2}|x| t+a|x|+\frac{1}{4 !}\left((t+2 a)^{3}-(2 a)^{3}\right)\right]^{p}
$$

(ii) for $p=2 n, n \in \mathbf{N}$,

$$
\mathbf{E}^{w}\left(\log ^{p} \psi_{t}(x)\right) \leq c_{2}^{p}\left[p+|x|(t+a) \frac{1}{4 !}+\left((t+2 a)^{3}-(2 a)^{3}\right)+\sqrt{p}\left((t|x|)^{3 / 4}\right)\right]^{p} \text {. }
$$

Proof. (i) Let $L_{p} \in C^{0}\left(\mathbf{R}^{+}\right)$be the convex hull of the function $\xi \mapsto \log ^{p} \xi$, by Jensen inequality

$$
\begin{aligned}
\mathbf{E}^{w}\left(\log ^{p} \psi_{t}(x)\right) & \leq \mathbf{E}^{w}\left(L_{p}\left(\psi_{t}(x)\right)\right) \leq L_{p}\left(\mathbf{E}^{w}\left(\log ^{p} \psi_{t}(x)\right)\right) \\
& \leq L_{p}\left(c e^{\frac{1}{2}|x| t+a|x|+\frac{1}{4^{\prime}}\left((t+2 a)^{3}-(2 a)^{3}\right)}\right)
\end{aligned}
$$

as $L_{p}$ is an increasing function. We note that $L_{p}(\xi)=\log ^{p} \xi$ for $\xi \geq \xi_{p}=\exp \{p-1\}$. Thus, when either $|x|$ or $t$ are large enough, we get the result.

(ii) For $p$ even function $\xi \mapsto \log ^{p} \xi$ is monotone decreasing for $\xi \in(0,1]$, monotone increasing for $\xi>1$ and concave for $\xi>\xi_{p}$. We bound separately the three regions. We have

$$
\begin{aligned}
\mathbf{E}^{w}\left(\chi_{\{\psi \leq 1\}} \log ^{p} \psi_{t}(x)\right) & \leq \mathbf{E}^{w}\left(\mathbf{E}_{x, t}^{\beta}\left(\log \psi_{0}\left(\beta_{0}\right)-\int_{0}^{t} d W_{s}\left(\beta_{s}\right)\right)\right)^{p} \\
& \leq\left[\mathbf{E}_{x, t}^{\beta}\left(\log \psi_{0}\left(\beta_{0}\right)\right)+\left\|\mathbf{E}_{x, t}^{\beta}\left(\int_{0}^{t} d W_{s}\left(\beta_{s}\right)\right)\right\|_{p}\right]^{p} \\
& \leq\left[a(1+|x|)+c \sqrt{p}\left((t|x|)^{1 / 2}+t^{3 / 4}\right)\right]^{p}
\end{aligned}
$$

where the last inequality is obtained by explicit computations of gaussian integrals. 
On the other hand

$$
\mathbf{E}^{w}\left(\chi_{\left\{1<\psi \leq \xi_{p}\right\}} \log ^{p} \psi_{t}(x)\right) \leq \log ^{p} \xi_{p} .
$$

Denote by $\tilde{\mathbf{E}}^{w}$ the expectation with respect to $d P^{w}$ conditioned to the event $\left\{\psi>\xi_{p}\right\}$; by Jensen inequality we finally have

$$
\begin{aligned}
\mathbf{E}^{w} & \left(\chi_{\left\{\psi>\xi_{p}\right\}} \log ^{p} \psi_{t}(x)\right) \leq P^{w}\left(\left\{\psi>\xi_{p}\right\}\right) \log \tilde{\mathbf{E}}^{w}\left(\psi_{t}(x)\right) \\
\leq & P^{w}\left(\left\{\psi>\xi_{p}\right\}\right) \log ^{p} \frac{\mathbf{E}^{w}\left(\psi_{t}(x)\right)}{P^{w}\left(\left\{\psi>\xi_{p}\right\}\right)} \\
\leq & \left.P^{w}\left(\psi>\xi_{p}\right\}\right)\left[\log c_{2}+\frac{1}{2}|x|(t+a)\right. \\
& \left.+\frac{1}{4 !}\left((t+2 a)^{3}-(2 a)^{3}\right)-\log P^{w}\left(\left\{\psi>\xi_{p}\right\}\right)\right]^{p},
\end{aligned}
$$

where we used the bound in (6.1) for $p=1$. Since for $\xi \in[0,1], \xi \log ^{p} \xi \leq(c / e)^{p}$, (6.5) follows from (6.7), (6.8) and (6.9).

We now bound from below the moments of $\log \psi_{t}(x)$ under the following restrictions: there exist $c_{1}, c_{2}>0$ such that for all $x \in \mathbf{R}, 1<c_{1} \leq \psi_{0}(x) \leq c_{2}$.

Proposition 6.3. Let $\psi_{t}(x)$ as in Proposition 2.1, then

(i) for $p=2 n+1, n \in \mathbf{N}$,

$$
\begin{aligned}
\mathbf{E}^{w}\left(\log ^{p} \psi_{t}(x)\right) \geq & \sqrt{p} ! c^{p}\left[|x|\left(t \wedge\left(2 x^{2}\right)\right)-\left(t \wedge\left(2 x^{2}\right)\right)^{3 / 2}\right. \\
& \left.+\theta\left(t-2 x^{2}\right) \cdot\left(t^{3 / 2}-|x|^{3}\right)\right]^{\frac{p-1}{2}}
\end{aligned}
$$

(ii) For $p=2 n, n \in \mathbf{N}$

$$
\mathbf{E}^{w}\left(\log ^{p} \psi_{t}(x)\right) \geq c_{1}^{p}\left((p !)^{3}\left(t^{2} x^{2}+t^{3}\right)^{p}\right)^{1 / 4}-c_{2}^{p} p !
$$

Proof. (i) Since $\log ^{p} \xi$ is increasing for $p$ odd, by Jensen inequality we have

$$
\begin{aligned}
& \mathbf{E}^{w} \log ^{p} \psi_{t}(x) \geq \mathbf{E}^{w}\left(\mathbf{E}_{x, t}^{\beta}\left(\log \psi_{0}\left(\beta_{0}\right)-\int_{0}^{t} d W_{s}\left(\beta_{s}\right)\right)\right)^{p} \\
& =\sum_{k=0}^{p} \frac{p !}{k !(p-k) !}\left(\mathbf{E}_{x, t}^{\beta} \log \psi_{0}\left(\beta_{0}\right)\right)^{p-k} \cdot \mathbf{E}^{w}\left(\mathbf{E}_{x, t}^{\beta}\left(-\int_{0}^{t} d W_{s}\left(\beta_{s}\right)\right)\right)^{k} \\
& \geq p ! ! c^{p}\left[\mathbf{E}_{x, t}^{\beta^{1}, \beta^{2}} \int_{0}^{t} d s C\left(\beta_{s}^{1}, \beta_{s}^{2}\right)\right]^{\frac{p-1}{2}} .
\end{aligned}
$$

The bound (6.10) can then be obtained after an explicit computation of the expectation in (6.12).

(ii) Let $X=\psi_{0}\left(\beta_{0}\right) \exp \left\{-M_{\beta}(t)\right\}$, following the same argument of (6.9), we have

$$
\begin{aligned}
\mathbf{E}_{x, t}^{\beta}\left(\chi_{\left\{X>\xi_{p}\right\}} \log ^{p} X\right) & \leq 2^{p-1}\left(\log ^{p} \mathbf{E}_{x, t}^{\beta}(X)+(p / e)^{p}\right) \\
& =2^{p-1}\left(\log ^{p} \psi_{t}(x)+(p / e)^{p}\right)
\end{aligned}
$$


and

$$
\mathbf{E}_{x, t}^{\beta}\left(\chi_{\left\{1 \leq X \leq \xi_{p}\right\}} \log ^{p} X\right) \leq \log ^{p} \xi_{p}=p-1
$$

Thus

$$
\begin{aligned}
\log ^{p} \psi_{t}(x) \geq & c_{1}^{p} \mathbf{E}_{x, t}^{\beta}\left(\chi_{\{X>1\}} \sum_{k=0}^{p} \frac{p !}{k !(p-k) !}\left(-M_{\beta}(t)\right)^{k} \log ^{p-k} \psi_{0}\left(\beta_{0}\right)\right)-c_{2}^{p} p ! \\
\geq & c_{1}^{p} \mathbf{E}_{x, t}^{\beta}\left(\chi_{\{X>1\}} \sum_{k \text { even }} \frac{p !}{k !(p-k) !}\left(-M_{\beta}(t)\right)^{k} \log ^{p-k} \psi_{0}\left(\beta_{0}\right)\right) \\
& -c_{2}^{p}\left(p !+\mathbf{E}_{x, t}^{\beta} \log ^{p} \psi_{0}\left(\beta_{0}\right)\right) .
\end{aligned}
$$

As $\{X>1\} \supset\left\{M_{\beta}(t)<0\right\}$, taking the expectation with respect to $d P^{w}$ of $(6.15)$ the result follows.

As far as $u_{t}(x)=-\partial_{x} \psi_{t}(x) / \psi_{t}(x)$ is concerned, we have the following

Proposition 6.4. Let $u_{t}(x)$ as in Theorem 2.2, then for almost all $t \in[0, T]$ and $\forall \delta>0$,

$$
\liminf _{x \rightarrow \infty} \frac{\left\|u_{t}(x)\right\|_{2}}{|x|^{\frac{1}{2}+\delta}}=0 .
$$

Proof. In Sect. 4 we obtained the identity

$$
\log \psi_{t}(x)=G_{t} * \log \psi_{0}(x)+\frac{1}{2} \int_{0}^{t} d s G_{t-s} * u_{s}^{2}(x)-\int_{0}^{t} G_{t-s} d W_{s}(x),
$$

taking the expectation with respect to $d P^{w}$, Proposition 6.2 implies

$$
\int_{0}^{t} d s G_{t-s} *\left\|u_{s}\right\|_{2}^{2}(x) \leq c\left(1+(t+a)|x|+(t+2 a)^{3}\right),
$$

from which (6.16) is derived.

\section{Concluding Remarks}

We conclude with some remarks on open problems.

In this paper we have not discussed at all the questions of uniqueness of the solution we have constructed. This is not a simple problem. The difficulty comes from the unboundedness of the space domain. In the unperturbed case (1.1) uniqueness was obtained by Hopf [7], by reducing the problem to the uniqueness of positive solutions of the heat equation. In our case this approach presents various difficulties. In Sect. 4 we proved that to a class of solutions of the linear equation (1.12) can be associated a class of solutions of the Burgers equation (1.5). The converse statement is not obvious as the application of Ito calculus requires the introduction of a regularization which in this direction is more difficult to remove. Furthermore the uniqueness problem for (1.12) is difficult due to the bad behavior of the random potential at infinity. On the other hand, in an unbounded domain, a naive attempt to obtain a Gronwall inequality 
for an appropriate norm of the solution of Eq. (1.5) fails due to the structure of the nonlinearity.

In the study of the stochastic Burgers equation a most relevant question is the behavior of the solutions when $\nu$ or $\varepsilon$ or both tend to zero. Here we have to distinguish different regimes. We consider the solution over a given time interval.

- $\nu$ finite, $\varepsilon \rightarrow 0$. The study of this case amounts essentially to the construction of a large deviation theory for the Burger equation. The main difficulties in the construction of such a theory are again connected to the infinite domain.

- $\varepsilon$ finite, $\nu \rightarrow 0$. A way to approach this problem at the formal level is to evaluate the Feynman-Kac formula by a Laplace type approximation. One obtains a formal expression which is the same one would obtain by solving the stochastically perturbed inviscid Burgers equation via the method of characteristics. This formal expression is a distribution valued process. This means that its square is not well defined and the mathematical interpretation of the stochastic inviscid equation is not apparent; some renormalization may be necessary. The relationship of these limit solutions, if they exist, to shock waves should be investigated. This problem has some similarity to that considered by Lax in [9].

- When $\varepsilon, \nu \rightarrow 0$, on the basis of heuristic arguments, the solution should converge to those of the unperturbed inviscid equation. However this conclusion may be false as the result may depend on the way the double limit is taken.

Acknowledgements. We thank R. Sénéor for useful discussions and the Ecole Polytechnique for hospitality at an early stage of this work, S. Olla for calling our attention to the Lax phenomenon and E. Presutti for helpful comments. This work was partially supported by EEC contract SC $1^{*} 0394$ $\mathrm{C}(\mathrm{EDB})$.

Note added in proof. After completing this work we received the following preprints, The Burgers Equation with a Noisy Force by H. Holden, T. Lindstrøm, B. Øksendal, J. Ubøe and T.-S. Zhang, where the stochastic Burgers equation is studied in the framework of white noise calculus; Stochastic Burgers Equation by G. Da Prato, A. Debussche, R. Temam where a detailed study on a finite space interval is made with a different approach.

\section{References}

1. Albeverio, S., Molchanov, S.A., Surgailis, D.: Stratified Structure of the Universe and the Burgers' Equation - A Probabilistic Approach. Preprint (1993)

2. Benzi, R., Jona-Lasinio, G., Sutera, A.: Stochastically Perturbed Landau-Ginzburg Equations. J. Stat. Phys. 55, 505-522 (1989)

3. Burgers, J.M.: The Nonlinear Diffusion Equation. Dordrecht: D. Reidel, 1974

4. Buttà, P.: Two dimensional Yang-Mills measure as a distribution valued brownian motion. SISSA Preprint 21/92/FM (1992)

5. Cole, J.D.: On a Quasi-Linear Parabolic Equation Occurring in Aerodynamics. Quart. Appl. Math. 9, 225-236 (1951)

6. Gihkman, I.I., Skorohod, A.V.: Stochastic Differential Equations. Berlin Heidelberg New York: Springer 1972

7. Hopf, E.: The Partial Differential Equation $u_{t}+u u_{x}=\mu u_{x x}$. Commun. Pure Appl. Math. 3, 201-230 (1950)

8. Ikeda, N., Watanabe, S.: Stochastic Differential Equations and Diffusion Processes. Amsterdam, Oxford, New York: North Holland Publishing, 1981

9. Lax, P.D.: The Zero Dispersion Limit, A Deterministic Analogue of Turbulence. Commun. Pure Appl. Math. 44, 1047-1056 (1991) 
10. Lighthill, M.J.: Viscosity Effects in Sound Waves of Finite Amplitude. In: Batchelor, G.K., Davies, R.M. (eds.) Surveys in Mechanics, Cambridge: Cambridge University Press, 1956, pp. 250-351

11. Molchanov, S.A.: Ideas in Theory of Random Media. Acta Appl. Math. 22, 139-282 (1991) and references therein

12. Mueller, C.: On the Support of Solutions to the Heat Equation with Noise. Stochastics and Stochastics Reports 37, 225-245 (1991)

13. Revuz, D., Yor, M.: Continuous Martingales and Brownian Motion. Berlin, Heidelberg, New York: Springer 1991

14. She, Z.S., Aurell, E., Frisch, U.: The Inviscid Burgers Equation with Initial Data of Brownian Type. Commun. Math. Phys. 148, 623-641 (1992)

15. Sinai, Ya.G.: Two Results Concerning Asymptotic Behavior of Solution of the Burgers Equation with Force. J. Stat. Phys. 64, 1-12 (1991)

16. Sinai, Ya.G.: Statistics of Shocks in Solutions of Inviscid Burgers Equation. Commun. Math. Phys. 148, 601-621 (1992)

17. Walsh, J.B.: An introduction to stochastic partial differential equations. In: Lecture Notes in Mathematics 1180. Berlin, Heidelberg, New York: Springer 1984

Communicated by Ya. G. Sinai 